\title{
ISING MODEL OF THIN FILM WITH RANDOM SURFACE FIELD*
}

\author{
Z. ONYSZKIEWICZ \\ Institute of Physics, A. Mickiewicz University \\ Matejki 48/49, 60-780 Poznań, Poland
}

(Received March 21, 1991)

\begin{abstract}
Thermodynamical properties of an Ising model of a ferromagnetic thin film deposited onto a ferromagnetic substrate of rough surface contacting the thin film were studied. The influence of the interaction between the substrate and the film as well as the effect of the substrate surface roughness were found to be describable in this model by a temperature dependent random surface field. Magnetization of the thin film was calculated for various temperatures at different assumed values of the substrate Curie temperature as well as for various surface roughness grades of the substrate and various couplings between the film and the substrate.
\end{abstract}

PACS numbers: $75.70 .-\mathrm{i}$

\section{Introduction}

Frequently in experiments aiming at the investigation of thin films we deal with films deposited on a bulk substrate which usually has a somewhat rough surface. However, most often in the theoretical description of such experiments the interaction between the film and the substrate as well as the effect of the substrate surface roughness are neglected. The main purpose of this work is to find a way to include these two factors and calculate their effect on the thermodynamical properties of the Ising model of a ferromagnetic thin film. To achieve this we apply the recently proposed statistical operator [1] for thin film systems and carry out the calculations within the molecular field approximation (MFA).

*Supported by the Project CPBP. 01.08 and the Department of Theoretical Physics, University of Salerno. 


\section{Model}

A Hamiltonian for our thin film is specified as follows $\left(s=\frac{1}{2}\right)$ :

$$
H=-\frac{1}{2} \sum K_{f_{r} f^{\prime}{ }^{\prime}} S_{f_{r}}^{z} S_{f^{\prime} r^{\prime}}^{z} .
$$

Here $f$ denotes the bidimensional position vectors of spins belonging to a given monoatomic layer. The summations always run over different sites. According to the Valenta [2] model the film is divided into $R$ monoatomic layers parallel to the planes (100) of a simple cubic lattice. The position of each monoatomic layer is given by the number $r=1,2, \ldots, R$. Our considerations are restricted to nearest neighbour interaction only. Let us assume that the thin film considered is deposited on a bulk ferromagnetic substrate sufficiently well described by the Ising model $\left(s=\frac{1}{2}\right)$ for an sc lattice and of the Hamiltonian

$$
H_{s}=-\frac{1}{2} \sum_{\boldsymbol{g} \neq g^{\prime}} I_{g g^{\prime}} S_{g^{z}}^{z} S_{g^{\prime}}^{z}
$$

where $g$ and $g^{\prime}$ are the position vectors of the spins in the substrate. Let us also assume that the film interacts with the substrate and this interaction may be described by a Heisenberg term of the form

$$
H_{I}(\xi)=-\frac{1}{2} \sum_{f g(r=1)} L(\xi) \boldsymbol{S}_{f} \cdot \boldsymbol{S}_{\boldsymbol{g}},
$$

where $\xi$ is the distance between the spins in a monoatomic layer of $r=1$ and their nearest neighbours in the substrate. Moreover, let

$$
L(\xi)=\left\{\begin{array}{l}
0 \text { for } \xi>a \\
\mathrm{~L} \text { for } \xi=a,
\end{array}\right.
$$

where $a$ is the smallest distance between these spins determined by the thin film and the substrate lattice symmetries. Equation (4) means that we take into account only the interaction between the nearest neighbours from the monoatomic layer of $r=1$ and from the substrate.

Finally we shall assume that the bulk substrate the film is deposited on has a rough surface which in our case means that $\xi$ can be treated as a random variable (see Fig. 1). Since in the substrate the spin positions at the sc lattice sites are favoured we assume the bimodal (sum of two $\delta$-functions) form of the probability distribution $p(\xi, c)$ of the random variable $\xi$ :

$$
p(\xi, c)=c \delta(\xi-a)+(1-c) \delta(\xi-2 a),
$$

where

$$
\int_{0}^{\infty} \mathrm{d} \xi p(\xi, c)=1
$$

For $c=0$ we have the case with no interaction between the thin film and the substrate whereas $c=1$ describes the case with this interaction but when the 


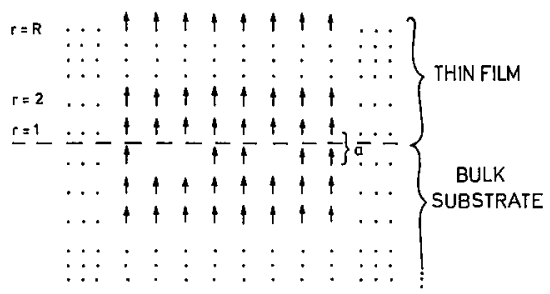

Fig. 1. Schematic diagram of ferromagnetic thin film deposited onto ferromagnetic substrate with rough surface.

substrate surface is even. Thus, the parameter $c$ can in a sense be treated as describing the grade of the substrate surface roughness.

In order to take into account the interaction of the thin film with the bulk substrate which may be treated ' as a thermostat for the film considered, we shall apply the recently proposed equilibrium statistical operator [1] suitable for description of physical situations similar to the one we are concerned with. In our case this statistical operator takes the following form:

$$
\varrho=\exp [\beta(F-H-V(\xi, \beta))],
$$

where

$$
V(\xi, \beta)=\operatorname{Tr}_{\mathrm{s}}\left[H_{I}(\xi) \exp \left(\beta\left(F_{\mathrm{s}}-H_{\mathrm{s}}\right)\right)\right]
$$

is an effective term describing the interaction between the thin film and the substrate and $\operatorname{Tr}_{\mathrm{s}}[\ldots]$ is the partial trace over the substrate states, $\beta=\left(k_{\mathrm{B}} T\right)^{-1}$ and

$$
F_{\mathrm{s}}=-\frac{1}{\beta} \ln \operatorname{Tr}\left[\exp \left(-\beta H_{s}\right)\right] .
$$

Substituting expression (3) into Eq. (7) under the assumption that

$$
\left|\left\langle s_{g}^{x}\right\rangle\right|,\left|\left\langle s_{g}^{y}\right\rangle\right| \ll\left|\left\langle s_{g}^{z}\right\rangle\right|,
$$

we come to

$$
V(\xi, \beta)=-H(\xi, \beta) \sum_{f, r=1} S_{f}^{z}
$$

where

$$
H(\xi, \beta)=\frac{1}{2} L(\xi)\left\langle S_{g}^{z}\right\rangle_{\mathrm{s}}
$$

and $\left\langle S_{g}^{z}\right\rangle_{\mathrm{s}}$ is the mean value of the substrate spin moment found from the formula

$$
\left\langle S_{\boldsymbol{g}}^{z}\right\rangle_{\mathrm{s}}=\operatorname{Tr}_{\mathrm{s}}\left[S_{\boldsymbol{g}}^{z} \exp \left(\beta\left(F_{\mathrm{s}}-H_{\mathrm{s}}\right)\right)\right] .
$$


Equation (10) implies that in the above approach the interaction between the film and the substrate with a rough surface may be taken into account through a temperature dependent random surface field $H(\xi, \beta)$.

Equation (6) leads to the following formula for the free energy:

$$
F(\xi)=-\frac{1}{\beta} \ln \operatorname{Tr}[\exp \{\beta(H+V(\xi, \beta))\}],
$$

which after averaging over the probability distribution (5) takes the form

$$
F=-\frac{1}{\beta} \int_{0}^{\infty} \mathrm{d} \xi p(\xi, c) \ln \operatorname{Tr}[\exp \{\beta(H+V(\xi, \beta))\}] .
$$

\section{Free energy}

Let us describe the thermodynamical properties of model (1) within the MFA. In order to achieve this we shall make use of the statistical operator (6) and the well-known variational principle for the free energy

$$
F(\xi) \leq \bar{F}(\xi)=F_{0}+\left\langle H-V(\xi, \beta)-H_{0}\right\rangle_{0},
$$

where

$$
\begin{aligned}
& \langle\ldots\rangle_{0}=\operatorname{Tr}\left[\ldots \varrho_{0}\right], \\
& \varrho_{0}=Z_{0}^{-1} \mathrm{e}^{-\beta H_{0}}, \\
& F_{0}=-\frac{1}{\beta} \ln Z_{0}
\end{aligned}
$$

and

$$
Z_{0}=\operatorname{Tr}\left[\mathrm{e}^{-\beta H_{0}}\right] .
$$

The operator $H_{0}$ is a trial Hamiltonian specified as follows:

$$
H_{0}=-\frac{1}{\beta} \sum_{f_{r}} y_{r} S_{f_{r}}^{z} \text {. }
$$

The variational parameters $y_{r}$ in (19) can be determined from the extremum conditions for the free energy $\bar{F}(\xi)$ :

$$
\frac{\partial \bar{F}(\xi)}{\partial y_{r}}=0 \quad(r=1,2, \ldots, R) .
$$

From Eqs. (14) to (20) we obtain

$$
f(\xi)=\sum_{r=2}^{R-1}\left[2\left\langle S_{r}^{z}\right\rangle^{2}+\frac{1}{2}\left\langle S_{r}^{z}\right\rangle\left(\left\langle S_{r-1}^{z}\right\rangle+\left\langle S_{r+1}^{z}\right\rangle\right)-t \ln 2 \cosh \frac{1}{2} y_{r}\right]+
$$




$$
\begin{aligned}
& +2\left\langle S_{1}^{z}\right\rangle^{2}+\frac{1}{2}\left\langle S_{1}^{z}\right\rangle\left\langle S_{2}^{z}\right\rangle-t \ln 2 \cosh \frac{1}{2} y_{1} \\
& +2\left\langle S_{R}^{z}\right\rangle^{2}+\frac{1}{2}\left\langle S_{R}^{z}\right\rangle\left\langle S_{R-1}^{z}\right\rangle-t \ln 2 \cosh \frac{1}{2} y_{R},
\end{aligned}
$$

where

$$
\begin{aligned}
& y_{r}=t^{-1}\left(4\left\langle S_{r}^{z}\right\rangle+\left\langle S_{r-1}^{z}\right\rangle+\left\langle S_{r+1}^{z}\right\rangle\right)(r=2,3, \ldots, R-1), \\
& y_{1}=t^{-1}\left(h(\xi, t)+4\left\langle S_{1}^{z}\right\rangle+\left\langle S_{2}^{z}\right\rangle\right), \\
& y_{R}=t^{-1}\left(4\left\langle S_{R}^{z}\right\rangle+\left\langle S_{R-1}^{z}\right\rangle\right)
\end{aligned}
$$

and

$$
\begin{aligned}
\left\langle S_{r}^{z}\right\rangle & =\frac{1}{2} \operatorname{th} \frac{1}{2} y_{r}, \\
\left\langle S_{1}^{z}\right\rangle & =\frac{1}{2} \operatorname{th} \frac{1}{2} y_{1}, \\
\left\langle S_{R}^{z}\right\rangle & =\frac{1}{2} \operatorname{th} \frac{1}{2} y_{R}, \\
f(\xi) & =(K N)^{-1} \bar{F}(\xi), \quad t=K^{-1} k_{\mathrm{B}} T, \\
K N & =\sum_{f \neq f} K_{f_{r} f^{\prime} r^{\prime}}, \quad h(\xi, t)=K^{-1} H(\xi, t) ;
\end{aligned}
$$

$N$ is the number of spins in a monoatomic layer. Substituting (21) into (14) we obtain

$$
\begin{aligned}
& f=\sum_{r=2}^{R-1}\left[2 x_{r}^{2}+\frac{1}{2} x_{r}\left(x_{r-1}+r_{r+1}\right)-t \ln 2 \cosh \frac{1}{2} y_{r}\right] \\
& +2 x_{R}^{2}+\frac{1}{2} x_{R} x_{R-1}-t \ln 2 \cosh \frac{1}{2} y_{R} \\
& +2 x_{1}^{2}+\frac{1}{2} x_{1} x_{2}-t c \ln 2 \cosh \left[(1 / 2 t)\left(h(t)+4 x_{1}+x_{2}\right)\right] \\
& -t(1-c) \ln 2 \cosh \left[(1 / 2 t)\left(4 x_{1}+x_{2}\right)\right],
\end{aligned}
$$

where

$$
\begin{aligned}
& h(t)=h_{0} m(t), \\
& m=2\left\langle S_{g}^{z}\right\rangle_{\mathrm{s}}, \quad h_{0}=\frac{1}{4} L K^{-1},
\end{aligned}
$$

and

$$
x_{r}=\frac{1}{2} \operatorname{th}\left[\frac{1}{2 t}\left(4 x_{r}+x_{r-1}+x_{r+1}\right)\right] \quad(r=2,3, \ldots, R-1),
$$



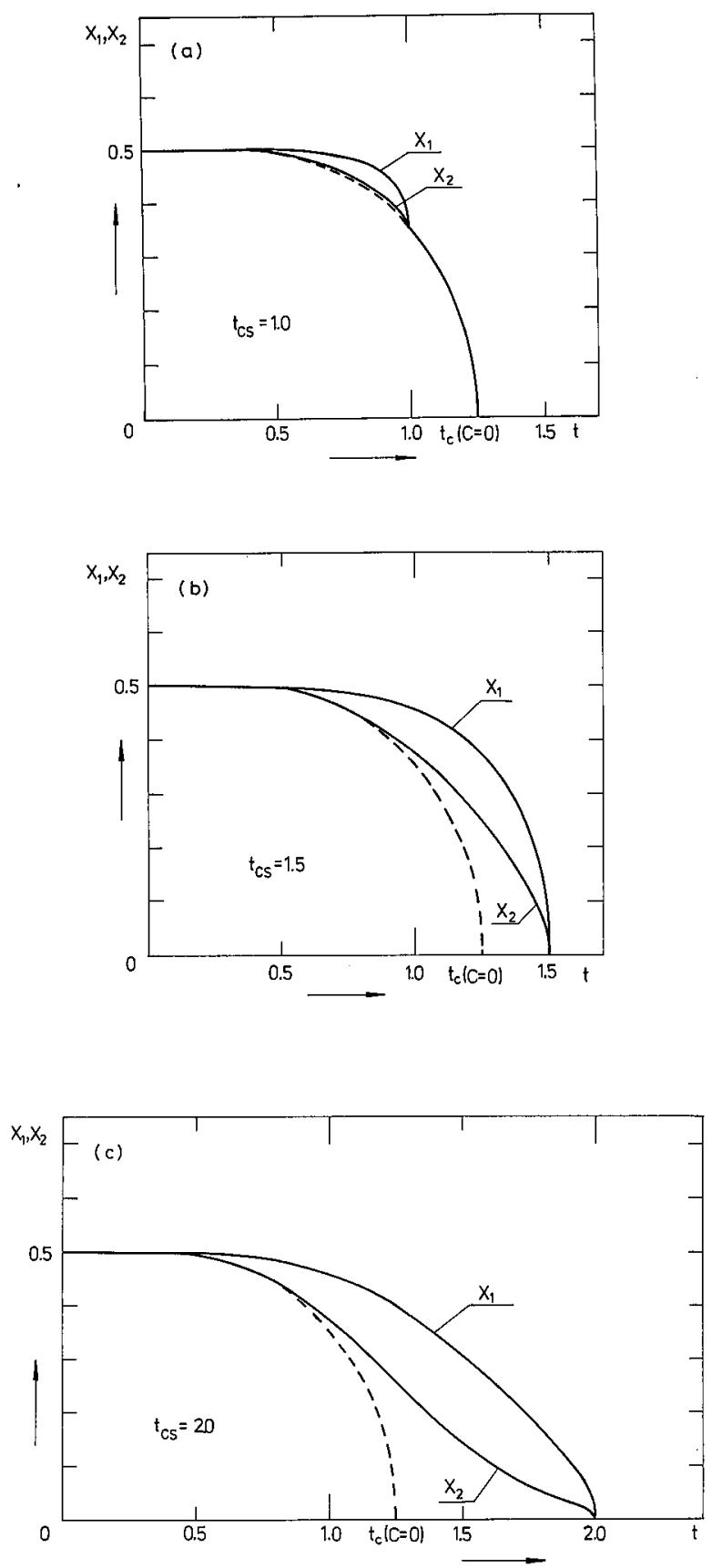

Fig. 2. Temperature dependence of magnetization $x_{1}$ and $x_{2}$ of monoatomic layers for $c=1$ ( $\longrightarrow$ ) and for $c=0(--$,$) as well as for different values of Curie$ temperature of substrate $t_{c s}\left(h_{0}=1\right)$. 


$$
\begin{aligned}
& x_{R}=\frac{1}{2} \operatorname{th}\left[\frac{1}{2 t}\left(4 x_{R}+x_{R-1}\right)\right], \\
& x_{1}=\frac{1}{2} \text { cth }\left[\frac{1}{2 t}\left(h(t)+4 x_{1}+x_{2}\right)\right]+\frac{1}{2}(1-c) \operatorname{th}\left[\frac{1}{2 t}\left(4 x_{1}+x_{2}\right)\right] .
\end{aligned}
$$

Mean magnetization $m$ of the substrate in MFA satisfies the equation

$$
m=\operatorname{th} \frac{t_{\mathrm{cs}}}{t} m
$$

where $t_{\mathrm{cs}}$ is the Curie temperature of the substrate.

Equations from (30) to (33) make a closed set permitting the calculation of magnetic properties of our thin film.

\section{Results and conclusion}

To illustrate our method and the way the interaction between the film and its substrate with a rough surface may be taken into account, we shall consider the simplest case of a thin film model of two monoatomic layers, $R=2$. The solutions of Eqs. (30-33) we obtained for this case are graphically illustrated in Figs. 2-4. Figure 2 presents the temperature (in relative units) dependence of magnetization $x_{1}$ and $x_{2}$ of monoatomic layers for different values of Curie temperature of the substrate $t_{\mathrm{cs}}$. Figure 3 shows the magnetization $x_{1}$ and $x_{2}$ of monoatomic layers

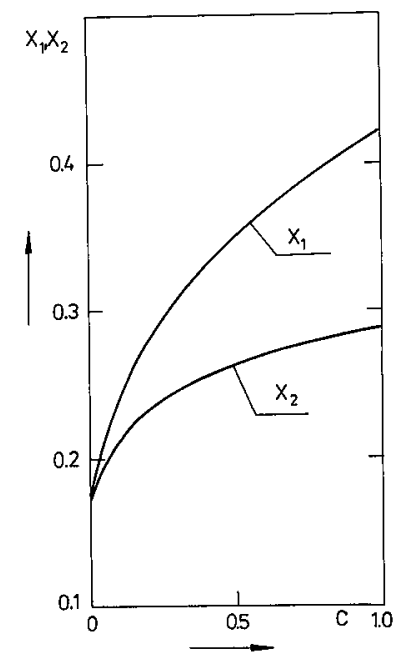

Fig. 3. Magnetization $x_{1}$ and $x_{2}$ of monoatomic layers versus parameter of probability distribution $c$ for $t_{c s}=3, h_{0}=1$ and $t=1.2$.

versus the parameter of probability distribution of $c$ which describes the grade of the substrate surface roughness. Finally, Fig. 4 presents the dependences of $x_{1}$ and 


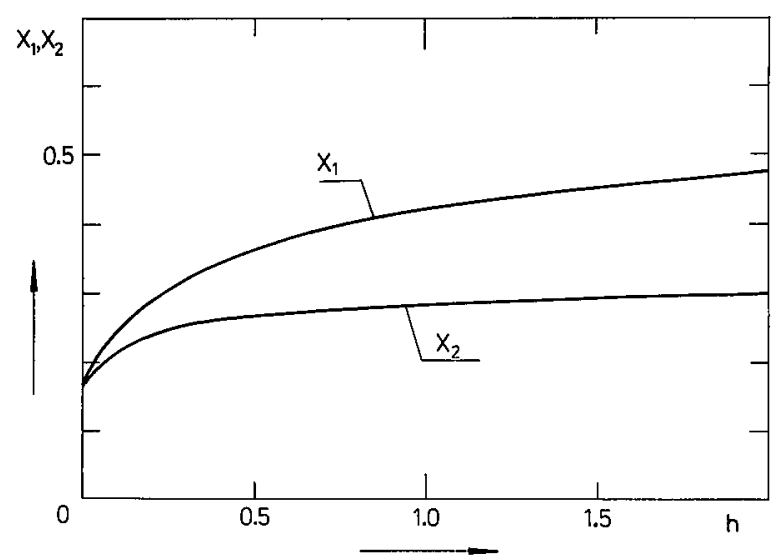

Fig. 4. Magnetization monoatomic layers $x_{1}$ and $x_{2}$ plotted against surface field $h$ for $t=1.2$ and $c=1$.

$x_{2}$ on the value of the parameter $h$ which for a given temperature is a measure of the degree of interaction with the substrate.

As follows from the above results, the interaction of a ferromagnetic thin film with its bulk ferromagnetic substrate and the grade of the substrate surface roughness may have an essential influence on the ferromagnetic film properties.

The Curie temperature $t_{c}$ of the film strongly depends on the Curie temperature $t_{\mathrm{cs}}$ of the substrate,

$$
t_{\mathrm{c}}= \begin{cases}t_{\mathrm{c}}(c=0) & \text { for } t_{\mathrm{cs}} \leq t_{\mathrm{c}}(c=0), \\ t_{\mathrm{cs}} & \text { for } t_{\mathrm{cs}}>t_{c}(c=0) .\end{cases}
$$

Obviously, these results are only illustrative in character and it would be difficult to verify them in experiment. We suppose that the influence of the substrate may essentially change the elementary excitation spectrum of the film which could be experimentally detected in spin wave resonance measurements. However, this is a different problem that would require a separate paper.

We believe that the approach proposed in this paper may be successfully applied in investigations of the substrate influence on the thermodynamical properties of nonmagnetic thin films and more complex thin film systems.

\section{Acknowledgement}

I am grateful to Professor L. de Cesare for his useful discussion.

\section{References}

[1] Z. Onyszkiewicz, Phys. Status Solidi B 150, 109 (1988).

[2] L. Valenta, Czech. J. Phys. 7, 127 (1957). 